\title{
HYPOELLIPTICITY OF CERTAIN DEGENERATE ELLIPTIC BOUNDARY VALUE PROBLEMS $\left({ }^{1}\right)$
}

\author{
BY
}

\section{YAKAR KANNAI}

\begin{abstract}
The concept of hypoellipticity for degenerate elliptic boundary value problems is defined, and its relation with the hypoellipticity of certain pseudo-differential operators on the boundary is discussed (for second order equations). A theorem covering smoothness of solutions of boundary value problems such as $a(x) \partial u / \partial n+b(x) u=f(x)$ for the Laplace equation is proved. An almost complete characterization of hypoelliptic boundary value problems for elliptic second order equations in two dimensions is given via analysis of hypoelliptic pseudo-differential operators in one variable.
\end{abstract}

1. The regularity of solutions of boundary value problems has been widely studied in the literature (see e.g. [4] , [5]), under "nondegeneracy" conditions. The oblique derivative problem

$$
\Delta u=0 \text { in } G \subset R^{n},
$$

$$
\partial u / \partial l=f \text { on } \partial G
$$

has also been investigated by many authors. It is usually assumed that the vector field $l$ is not characteristic to that submanifold of $\partial G$ on which $l$ is tangential to $\partial G$. Then the pseudo-differential operator mapping $\left.u\right|_{\partial G}$ to $\partial u / \partial l$ is of principal type, and the addition of lower order terms in (1.2) plays no significant role in the theory [6]. This paper was motivated by the desire to discuss regularity properties of solutions of the problem where (1.2) is replaced by

$$
a \partial u / \partial l+b u=f \text { on } \partial G
$$

where $a$ might vanish somewhere. This could be done by transforming the boundary value problem (as in [6], [14]) to a problem concerning hypoellipticity of a certain pseudo-differential operator on $\partial G$. It turns out that this ap-

Received by the editors September 11, 1974. $47 \mathrm{E} 05$.

AMS (MOS) subject classifications (1970). Primary 35H05, 35J25; Secondary 35S05,

Key words and phrases. Boundary value problems, elliptic equations, hypoelliptic pseudo-differential operators, symbols, ordinary pseudo-differential operators.

(1) This work was partially supported by NSF Grant GP-35347X. 
proach yields quick results concerning smoothness of boundary value problems.

In $\$ 2$ we define precisely what we mean by hypoellipticity of a boundary value problem and prove that for (most) second order elliptic operators this is equivalent to the hypoellipticity of a certain pseudo-differential operator on $\partial G$. In $\S 3$ we exhibit a class of hypoelliptic operators arising naturally from consideration of (1.3). This class might also be of independent interest. In §4 we give an almost complete characterization of hypoelliptic boundary problems for two-dimensional problems (and second order equations). This is achieved by extending the results of [3], [11] from one-dimensional differential operators to one-dimensional pseudo-differential operators. For this extension a result enabling us to disregard all but finitely many terms in the asymptotic expansion of the pseudo-differential operator (Lemma 4.2) and an extension of the "stationary phase" principle to a case where there are singularities (Lemma 4.4) are important.

Our results include as special cases the results of S. Ito [10] and K. Hayashida [2] who proved smoothness of the solution $u$ of the problem

$$
L u=f \text { in } G, \alpha(x) \partial u / \partial n+\beta(x) u=0 \text { on } \partial G,
$$

where $L$ is a real second order elliptic operator and $\alpha \geqslant 0$. Ito assumes $\alpha+\beta$ $>0$. Hayashida makes a different assumption, whose meaning is analyzed in §3, Example 3. Note that Ito and Hayashida are concerned also with existence, whereas we are mostly concerned with smoothness.

The reduction of the boundary value problem to a problem involving a pseudo-differential operator, the discussion of the two-dimensional case, and some portions of $\$ 3$, can be extended to higher order operators; the computations, though, become much more involved.

I am very much indebted to Professor E. Shamir for suggesting Lemma 4.1 to me, and to Professors R. Beals, H. Brézis and C. Fefferman for helpful conversations.

2. Let $G$ be a bounded open subset of $R^{n}$ with $C^{\infty}$ boundary $\partial G$. Near $\partial G, G$ is of the form $\partial G \times(0,1)$; we suppose a normal coordinate $x_{n}$ is chosen near $G$, and we denote points of $G$ near $\partial G$ by $\left(x^{\prime}, x_{n}\right)$, and we set $u_{k}=$ $D_{n}^{k} u\left(x^{\prime}, 0\right), k=0,1, \ldots$. (In this paper $D_{j}=(1 / i)\left(\partial / \partial x_{j}\right)$.) Let $U_{1}, \ldots, U_{l}$ be a finite open covering of $\partial G$ with $U_{i}$ diffeomorphic to the unit sphere in $R^{n-1}, 1 \leqslant i \leqslant l, h_{i}$ being the diffeomorphism and let $\varphi_{1}, \ldots, \varphi_{l}$ be a partition of unity subordinate to the covering $\left\{U_{i}\right\}_{i=1, \ldots, l}$. For $r, s$ reals we say that $u \in D^{\prime}(G)$ is in $H_{r, s}(G)$ if each of the distributions $v_{i}$ defined by

$$
v_{i}=\varphi_{i}\left(x^{\prime}\right) w\left(x_{n}\right) u \circ h_{i}
$$


is in $H_{r, s}\left(\bar{R}^{+}\right)$and $v=u\left(1-\Sigma_{i=1}^{l} \varphi_{i}\left(x^{\prime}\right) w\left(x_{n}\right)\right) \in H_{r, s}\left(R^{n}\right)$. Here $w \in C_{0}^{\infty}(-1,1)$ with $w \equiv 1$ near 0 and the spaces $H_{r, s}\left(R^{n}\right), H_{r, s}\left(\bar{R}^{+}\right)$are those introduced in [5, $\S 2.5] . H_{r, s}(G)$ is a Hilbert space with the norm

$$
\left(\|u\|_{r, s}\right)^{2}=\sum_{i=1}^{l}\left(\left\|v_{i}\right\|_{r, s}\right)^{2}+\left(\|v\|_{r, s}\right)^{2} .
$$

We refer to [5] for discussion of the $H_{r, s}$ spaces. It is important to note that if $u \in H_{m, s}(G)$ where $m$ is a positive integer, then the "Cauchy data" $u_{j}\left(x^{\prime}\right)$ are well-defined elements of $H_{(m+s-j-1 / 2)}(\partial G)$ for $0 \leqslant j \leqslant m-1$. (Here $H_{t}(\partial G)$ is the usual Sobolev space.) Let now $P(x, D)$ be an elliptic differential operator of order $m$ defined on $\operatorname{cl}(G)$ with $C^{\infty}$ coefficients, and let $\left\{B_{i j}\left(x^{\prime}, D^{\prime}\right)\right.$; $i=1, \ldots, k ; j=0, \ldots, m-1$ \} be (classical) pseudo-differential operators defined on $\partial G$, with $C^{\infty}$ coefficients. We say that the boundary value problem

$$
\begin{gathered}
P(x, D) u=f \text { in } G, \\
\sum_{j=0}^{m-1} B_{i j}\left(x^{\prime}, D^{\prime}\right) u_{j}=g_{i} \text { on } \partial G, \quad i=1, \ldots, k,
\end{gathered}
$$

is hypoelliptic if for $u \in H_{m, s}(G), f \in C^{\infty}(\mathrm{cl}(G))$ and $g_{i} \in C^{\infty}(V)$ we have $u \in C^{\infty}(G \cup V)$, where $V$ is an arbitrary relatively open subset of $\partial G$.

A few comments about this definition are not out of order. The ellipticity of $P$ implies that

$$
u_{m}\left(x^{\prime}\right)=\sum_{j=0}^{m-1} C_{j}\left(x^{\prime}, D^{\prime}\right) u_{j}\left(x^{\prime}\right)
$$

where $C_{j}\left(x^{\prime}, D^{\prime}\right), j=0, \ldots, m-1$, are differential operators tangential to $\partial G$. Hence any boundary differential operator can be represented in the form $\Sigma_{j=0}^{m-1} B_{j}\left(x^{\prime}, D^{\prime}\right) u_{j}$. In [4] hypoellipticity of the boundary value problem $P(D) u=0$ in $G, B_{i}(D) u=0$ on a planar portion of $\partial G$ was defined as the requirement that every classical solution of the problem be $C^{\infty}$. The restriction to homogeneous problems seems to be too weak for problems with nonconstant coefficients. Moreover, all $C^{2}$ solutions of the problem $\left(D_{1}^{2}+D_{2}^{2}\right) u=f$ in $x_{2}>0,\left[x_{1} D_{1}+(3 / 2) i\right] u=g$ on $x_{2}=0$ are $C^{\infty}$ if $f$ and $g$ are, but $u=$ $\operatorname{Re}\left(x_{1}+i x_{2}\right)^{3 / 2}$ is a nonsmooth solution of the problem with $f=g=0$, and thus it would be counter-intuitive to name this boundary value problem hypoelliptic. On the other hand, the assumption that $u \in H_{m, s}(G)$ for some $s$ is quite weak; compare [5, p. 256].

Let now $m=2$ and let

$$
P(x, D) u=\sum_{j, k=1}^{n} a_{j k}(x) D_{j} D_{k} u+\sum_{j=1}^{n} b_{j}(x) D_{j} u+c(x) u
$$


be a representation of $P$ in $h_{i}^{-1}\left(U_{i}\right)$ for some $i$, where $a_{j k}=a_{k j}$. We make now the following assumption (the so-called root condition): The quadratic equation in $t$,

$$
\sum_{j, k=1}^{n-1} a_{j k} \xi_{j} \xi_{k}+\sum_{j=1}^{n-1} a_{j n} \xi_{j} t+\sum_{k=1}^{n-1} a_{n k} \xi_{k} t+a_{n n} t^{2}=0
$$

has precisely one root $t^{\prime}\left(=t^{\prime}(x, \xi)\right)$ with positive imaginary part and one root $t^{\prime \prime}$ with negative imaginary part, for all $x$ in $h_{i}^{-1}\left(U_{i}\right)$ and all real nonzero vectors $\xi^{\prime}=\left(\xi_{1}, \ldots, \xi_{n-1}\right)$. (This assumption is always fulfilled if $n>2$; if $n=2$ it is stronger than ellipticity and weaker than the reality assumption of [2].) Following $[6, \S 2.2]$ we assume that $P$ is defined and is elliptic in a larger open set $H$ with $\operatorname{cl}(G) \subset H$, and let $T$ be a parametrix for $P$ in $H$, i.e., $T P=I+K$ where $K$ is a smoothing operator. For any $v \in H_{0, s}(G)$ denote by $v^{0} \in E^{\prime}(H)$ the distribution obtained by extending $v$ to be equal to 0 outside $\mathrm{cl}(G)$ (compare $[5$, p. 54]). Denoting by $\delta$ the surface measure on $\partial G$ we obtain

$$
P u^{0}=(P u)^{0}+i^{-1} \sum_{j=0}^{1} \sum_{l=0}^{1-j} P_{j+l+1} D_{n}^{j}\left(u_{l} \delta\right)
$$

for $u \in H_{2, s}(G)$, where $P_{j}=P_{j}\left(x^{\prime}, D^{\prime}\right)$ are determined by $P=\Sigma_{j=0}^{m} P_{j} D_{n}^{j}$, order $\left(P_{j}\right)$ $=m-j$. Applying $T$ to (2.5) we find

$$
u^{0}+K u^{0}=T(P u)^{0}+i^{-1} \sum_{j=0}^{1} \sum_{l=0}^{1-j} T P_{j+l+1} D_{n}^{j}\left(u_{l} \delta\right) .
$$

If $P u \in C^{\infty}(\mathrm{cl}(G))$ then the restriction of $T(P u)^{0}$ to $\partial G$ is a $C^{\infty}$ function. $K u^{0}$ is obviously smooth in all of $H$. Setting

$$
Q_{l} v=\left.\left(i^{-1} \sum_{j=0}^{1-l} T P_{j+l+1} D_{n}^{j}(v \delta)\right)\right|_{\partial G}
$$

for $v \in D^{\prime}(\partial G)$, we get

$$
u_{0}-Q_{0} u_{0}-Q_{1} u_{1}=w\left(x^{\prime}\right) \in C^{\infty}(\partial G) .
$$

According to [6] the pseudo-differential operator $Q_{1}$ is actually elliptic. The operator $R=Q_{1}^{-1}\left(I-Q_{0}\right)$ is a pseudo-differential operator with symbol

$$
r=t^{\prime}\left(x^{\prime}, 0 ; \xi^{\prime}\right)+s\left(x^{\prime}, \xi^{\prime}\right)
$$

where $s$ is a symbol of order zero.

THEOREM 2.1. Let $P(x, D)$ be an elliptic second order differential operator satisfying the root condition, let $B_{0}, B_{1}$ be (tangential) pseudo-differential operators defined on $\partial G$ and let $R$ be as above. Then the boundary value problem $P(x, D) u=f$ in $G, B_{0} u_{0}+B_{1} u_{1}=g$ on $\partial G$ is hypoelliptic if and only if the operator $L=B_{0}+B_{1} R$ is a hypoelliptic operator on the manifold $\partial G$. 
Proof. It follows from (2.8) that

$$
g=B_{0} u_{0}+B_{1} Q_{1}^{-1}\left(I-Q_{0}\right) u_{0}-B_{1} Q_{1}^{-1} w
$$

or

$$
g+B_{1} Q_{1}^{-1} w=L u_{0}
$$

Thus $u_{0} \in C^{\infty}(\partial G)$ if $L$ is hypoelliptic. But then $u_{1}=R u_{0}-Q_{1}^{-1} w$ is also in $C^{\infty}(\partial G)$. That $u \in C^{\infty}(\operatorname{cl}(G))$ follows then by applying Theorem 2.14 of [6] to $(2.6)$.

Conversely, suppose that $L$ is not hypoelliptic in a relatively open subset $V$ of $\partial G$. Let $u_{0} \in H_{s}(\partial G)$ satisfy $u_{0} \notin C^{\infty}(V), L u_{0} \in C^{\infty}(\partial G)$, for some $s$ (such $u_{0}$ clearly exists). The Dirichlet problem is elliptic (in the sense of [5]) for $P(x, D)$ (by the root condition). Applying Theorem 10.4.1 of [5] we construct a distribution $u \in H_{m, s}(G)$ satisfying $P u=0$ in a neighborhood $W \subset G$ with $\operatorname{cl}(W) \cap \partial G=V$ and $\left.u\right|_{V}=u_{0}$. Let $v$ be an extension of $\left.u\right|_{\operatorname{cl}(W)}$ to a function which is $C^{\infty}$ in $\operatorname{cl}(G) \backslash W$. Applying (2.8) we find that $w\left(x^{\prime}\right)=v_{0}-Q_{0} v_{0}-$ $Q_{1} v_{1}$ is a $C^{\infty}$ function on $\partial G$ and thus $B_{0} v_{0}+B_{1} v_{1}=L v_{0}-B_{1} Q_{1}^{-1} w$. But $L$ is pseudo-local and $\left.v_{0}\right|_{V}=\left.u_{0}\right|_{V}$, imply that $L v_{0} \in C^{\infty}(\partial G)$. Thus the boundary value problem $P v=f, B_{0} v_{0}+B_{1} v_{1}=g$ is not hypoelliptic.

Note that if the pseudo-differential operator $L$ is hypoelliptic then it has a finite-dimensional kernel in $C^{\infty}(\partial G)$. If its adjoint $L^{*}$ is also hypoelliptic then $L$ has a finite index. In many cases its index can be computed from its symbol [8]. One could then use the above reduction of a boundary value problem in $G$ to a pseudo-differential problem on $\partial G$ for investigating existence problems too.

If the symbols of $B_{0}, B_{1}$ are $b_{0}\left(x^{\prime}, \xi^{\prime}\right), b_{1}\left(x^{\prime}, \xi^{\prime}\right)$, respectively, then the symbol of $L$ is

$$
\begin{aligned}
q\left(x^{\prime}, \xi^{\prime}\right) \sim & b_{0}\left(x^{\prime}, \xi^{\prime}\right)+\sum_{\alpha} b_{1}^{(\alpha)}\left(x^{\prime}, \xi^{\prime}\right) i^{-|\alpha|_{r}} r_{(\alpha)}\left(x^{\prime}, \xi^{\prime}\right) \\
= & b_{0}\left(x^{\prime}, \xi^{\prime}\right)+\sum_{\alpha} b_{1}^{(\alpha)}\left(x^{\prime}, \xi^{\prime}\right) i^{-|\alpha|_{t}^{\prime}}\left({ }_{(\alpha)}\left(x^{\prime}, 0 ; \xi^{\prime}\right)\right. \\
& +\sum_{\alpha} b_{1}^{(\alpha)}\left(x^{\prime}, \xi^{\prime}\right) i^{-|\alpha|_{s}}\left(x^{\prime}, \xi^{\prime}\right)
\end{aligned}
$$

where $\alpha$ runs over all $(n-1)$-dimensional multi-indices, and as usual

$$
P_{(\beta)}^{(\alpha)}(x, \xi)=\left(i D_{x}\right)^{\beta}\left(i D_{\xi}\right)^{\alpha} p(x, \xi) .
$$

Note that $q\left(x^{\prime}, \xi^{\prime}\right)$ is just the characteristic function of the boundary value problem as defined in [4] if $P, B_{i}$ have constant coefficients and the boundary is planar. 
3. In this section we shall prove the hypoellipticity of certain degenerate pseudo-differential operators of order 1 and we shall apply them (using Theorem 2.1) for the study of boundary value problems.

It will be convenient to discuss the local question of hypoellipticity in Euclidean space and not in $\partial G$. Let thus $H$ be an open subset of $R^{q}$. The following is a partial generalization of Theorem 3 in [11].

THEOREM 3.1. Let

$$
q(x, \xi)=a(x) e(x, \xi)+b(x, \xi)
$$

where

$$
\begin{aligned}
& a(x) \in C^{\infty}(H) \text { and } a(y)=0, \\
& y \in H \text { implies }\left(D_{j} a\right)(y)=0, \quad j=1, \ldots, q .
\end{aligned}
$$

Assume further that $b(x, \xi), e(x, \xi) \in C^{\infty}\left(H \times\left(R^{q} \backslash\{0\}\right)\right)$ and that for all $(x, \xi) \in H \times\left(R^{q} \backslash\{0\}\right)$,

$$
\begin{gathered}
|e(x, \xi)| \leqslant C_{1}(1+|\xi|), \\
\left|e_{(\beta)}^{(\alpha)}(x, \xi)\right| \leqslant C_{2, \alpha, \beta}(1+|e(x, \xi)|)(1+|\xi|)^{-|\alpha| \rho+|\beta| \delta}
\end{gathered}
$$

for all multi-indices $\alpha, \beta$, where $\rho$ and $\delta$ are fixed constants satisfying $1 \geqslant \rho>$ $1 / 2 \geqslant \delta \geqslant 0$,

$$
\left|b_{(\beta)}^{(\alpha)}(x, \xi)\right| \leqslant C_{3, \alpha, \beta}(1+|\xi|)^{-\rho|\alpha|+\delta|\beta|}
$$

for all $\alpha_{s} \beta$,

$$
|a(x) e(x, \xi)|+|b(x, \xi)| \geqslant C_{5}>0 \text { if }|\xi|>C_{5}^{-1} \text {, }
$$

and

$$
\left|r_{1}(x, \xi)+\dot{u}_{2}(x, \xi)\right| \leqslant C_{6}\left(r_{1}(x, \xi)^{+}+\left|r_{2}(x, \xi)\right|\right) \text {, }
$$

where

$$
r_{1}(x, \xi)+i r_{2}(x, \xi)=a(x) e(x, \xi) / b(x, \xi),
$$

$r_{j}(x, \xi)$ is real, $j=1,2$, and $t^{+}=\max (t, 0)$.

Then there exists a constant $C_{7}>0$ such that for all $x \in H$

$$
|q(x, \xi)| \geqslant C_{7} \quad \text { if }|\xi|>C_{5}^{-1}
$$

and for every pair $(\alpha, \beta)$ of multi-indices and compact subset $K$ of $H$ there exists a constant $C_{8, \alpha, \beta, K}$ such that for all $x \in K,|\xi|>C_{5}^{-1}$,

$$
\left|q_{(\beta)}^{(\alpha)}(x, \xi)\right| \leqslant C_{8, \alpha, \beta, K}|q(x, \xi)|(1+|\xi|)^{-|\alpha| \rho+|\beta| / 2} .
$$


It follows from Theorem 4.2 of [7] that the pseudo-differential operator $q(x, D)$ is hypoelliptic. (Its adjoint is also hypoelliptic and the results of [8] are applicable.)

Proof. We shall denote from now on by $C$ any constant which is independent of $x \in K$ where $K$ is a compact subset of $H$ and $|\xi|>C_{5}^{-1}$. Note first that

$$
|b(x, \xi)| \leqslant C|q(x, \xi)| \text {. }
$$

Indeed, (3.9) is certainly true if $b(x, \xi)=0$. Otherwise

$$
q(x, \xi)=\left(r_{1}(x, \xi)+i r_{2}(x, \xi)+1\right) b(x, \xi) .
$$

If $\left|r_{1}+1\right| \leqslant 1 / 2$ then $r_{1}<-1 / 2$ and by (3.6) $1 / 2 \leqslant\left|r_{1}\right| \leqslant C\left|r_{2}\right|$, so that $\left|r_{2}\right| \geqslant C$ $>0$ and $|b| \leqslant|q| /\left|r_{2}\right| \leqslant C|q|$. If $\left|r_{1}+1\right| \geqslant 1 / 2$ then $|b| \leqslant 2|q|$, and thus (3.9) is always true. But then $|a e| \leqslant|q|+|b|$ implies also that

$$
|a(x) e(x, \xi)| \leqslant C|q(x, \xi)| \text {. }
$$

Estimate (3.7) thus follows from (3.9) and (3.11) together with (3.5). Taking now (3.4) into account, we see that we have to worry only about derivatives of $a e$ in proving (3.8). In estimating $x$-derivatives of $a$, the following generalization of a well-known [2], [13] property of nonnegative functions is useful:

LEMMA 3.2. Let $a(x) \in C^{2}(H)$ and assume that $a(x)$ has no simple zeros. Then for every compact subset $K$ of $H$ there exists a constant $C(K)$ such that for all $x \in K$ and $1 \leqslant j \leqslant q$,

$$
\left|\left(D_{j} a\right)(x)\right| \leqslant C|a(x)|^{1 / 2}
$$

Proof. It suffices to show that $\left|\left(D_{1} a\right)(x)\right| \leqslant C|a(x)|^{1 / 2}$. Let $L$ be a compact subset of $H$ containing $K$ in its interior, and let $\delta=\operatorname{dist}(K, H N)>0$. Set

$$
M=\max _{x \in L}\left\{\left|D_{1}^{2} a(x)\right|,|a(x)| / \delta^{2}\right\}
$$

For a given $y \in K$, set $\beta=|a(y)|^{1 / 2} / M^{1 / 2}$, and consider the function $f(s)=$ $f\left(y_{1}+s, y_{2}, \ldots, y_{n}\right)$. Then $f(s)$ is $C^{2}$ in the interval $|s| \leqslant \beta$, with $\left|f^{\prime \prime}(s)\right| \leqslant M$ in that interval and $\beta=(|f(0)| / M)^{1 / 2}$. If $\left|f^{\prime}(0)\right|$ were greater than $2 \beta M$, then it would follow from a form of the implicit function theorem given in [13, p. 346] that the interval $|s| \leqslant \beta$ contains one point $s_{0}$ where $f$ vanishes and $f^{\prime}$ does not. Thus $a$ would have a simple zero at the point $\left(y_{1}+s_{0}, y_{2}, \ldots, y_{n}\right) \in H$, a contradiction. Hence

$$
\left|D_{1} a(y)\right|=\left|f^{\prime}(0)\right| \leqslant 2 \beta M=2 M^{1 / 2}|a(y)|^{1 / 2} .
$$

END Of Proof of Theorem 3.1. According to Leibnitz's rule, 


$$
\begin{aligned}
\left|(a e)_{(\beta)}^{(\alpha)}(x, \xi)\right| & =\left|\sum_{0 \leqslant \gamma<\beta}\left(\begin{array}{l}
\beta \\
\gamma
\end{array}\right) a_{(\gamma)}(x) e_{(\beta-\gamma)}^{(\alpha)}(x, \xi)\right| \\
\leqslant C\left[|a(x)|\left|e_{(\beta)}^{(\alpha)}(x, \xi)\right|+\sum_{j=1}^{q}\left|D_{j} a(x)\right|\right. & \sum_{|\mu|=|\beta|-1}\left|e_{(\mu)}^{(\alpha)}(x, \xi)\right| \\
& \left.+\sum_{|\mu|<|\beta|-2}\left|e_{(\mu)}^{(\alpha)}(x, \xi)\right|\right] .
\end{aligned}
$$

The last term in the right-hand side of (3.14) can be estimated (due to (3.2) and (3.3)) by

$$
\begin{aligned}
C[1+|e(x, \xi)|](1+|\xi|)^{-|\alpha| \rho+|\beta| \delta-2 \delta} & \leqslant C(1+|\xi|)^{-|\alpha| \rho+|\beta| \delta-2 \delta+1} \\
& \leqslant C(1+|\xi|)^{-|\alpha| \rho+|\beta| / 2} .
\end{aligned}
$$

The middle sum can be estimated (applying (3.12), (3.2), (3.3) and (3.11)) by

$$
\begin{aligned}
& C|a(x)|^{1 / 2}(1+|e(x, \xi)|)(1+|\xi|)^{-|\alpha| \rho+|\beta| \delta-\delta} \\
& \leqslant C(1+|\xi|)^{-|\alpha| \rho+|\beta| \delta}+C|a(x) e(x, \xi)|^{1 / 2}|e(x, \xi)|^{1 / 2} \\
& \cdot(1+|\xi|)^{-|\alpha| \rho+|\beta| \delta-\delta} \\
& \leqslant C(1+|\xi|)^{-|\alpha| \rho+|\beta| \delta}+C|q(x, \xi)|^{1 / 2}(1+|\xi|)^{-|\alpha| \rho+|\beta| \delta+1 / 2-\delta} \\
& \leqslant C(1+|\xi|)^{-|\alpha| \rho+|\beta| \delta}+C|q(x, \xi)|^{1 / 2}(1+|\xi|)^{-|\alpha| \rho+|\beta| / 2}
\end{aligned}
$$

The first sum can be estimated, using (3.3) and (3.11) once more, by

$$
\begin{aligned}
& C\left[(1+|\xi|)^{-|\alpha| \rho+|\beta| \delta}+|a(x) e(x, \xi)|(1+|\xi|)^{-|\alpha| \rho+|\beta| \delta}\right] \\
& \leqslant C\left[(1+|\xi|)^{-|\alpha| \rho+|\beta| \delta}+|q(x, \xi)|(1+|\xi|)^{-|\alpha| \rho+|\beta| \delta}\right] .
\end{aligned}
$$

Applying (3.7) and $\delta \leqslant 1 / 2$, we get from (3.12)-(3.17) that

$$
\left|(a e)_{(\beta)}^{(\alpha)}(x, \xi)\right| \leqslant C|q(x, \xi)|(1+|\xi|)^{-|\alpha| \rho+|\beta| / 2}
$$

and Theorem 3.1 is proved.

EXAMPLE 1. $a$ and $b$ satisfy (3.1) and (3.4) respectively. $e(x, \xi) \equiv \mid \xi b$ $\exists \epsilon>0$ such that $|\arg [a(x) / b(x, \xi)]| \leqslant \pi-\epsilon$, and $|a(x)|+|b(x, \xi)|>0$. Example 1 is only a slight modification of the examples discussed at the end of $\$ 4$ in [7]. A more substantial application of Theorem 2.1 is

EXAMPLE 2. $a$ and $b$ satisfy (3.1) and (3.4) respectively, $e(x, \xi)=$ $\xi_{1}^{3}\left(\xi_{1}^{2}+\xi_{2}^{2}\right)^{-1}(q=2), \exists \epsilon>0$ such that $\operatorname{larg}[a(x) / b(x, \xi)] \mid \leqslant \pi-\epsilon$ and assume that (3.5) is satisfied. A simple computation shows that (3.3) is satisfied with $\rho=2 / 3, \delta=0$.

EXAMPLE 3. Let $\partial u / \partial n$ be the (outward) conormal derivative associated with an operator $P$ satisfying the conditions of Theorem 2.1 and consider, as in [2], [10], the boundary operator $c\left(x^{\prime}\right) \partial / \partial n+d\left(x^{\prime}\right)$. Then in a local coordinate 
system we can write

$$
\frac{\partial u}{\partial n}=-\sum_{j=1}^{n} a_{j n}\left(x^{\prime}, 0\right) \frac{\partial}{\partial x_{j}}=-\sum_{j=1}^{n-1} a_{j n}\left(x^{\prime}, 0\right) \frac{\partial}{\partial x_{j}}-a_{n n} \frac{\partial}{\partial x_{n}} .
$$

The ellipticity of $L$ implies that $a_{n n} \neq 0$ and we can assume that $a_{n n}>0$. Solving (2.4) we find that

$$
t^{\prime}=\left(-2 \sum_{j=1}^{n-1} a_{j n} \xi_{j}+A\left(x^{\prime}, \xi^{\prime}\right)\right)\left(2 a_{n n}\right)^{-1}
$$

where $A\left(x^{\prime}, \xi^{\prime}\right)$ is the square root with positive imaginary part of the discriminant of the quadratic equation (2.4). In our case

$$
b_{0}\left(x^{\prime}, \xi^{\prime}\right)=d\left(x^{\prime}\right)-i c\left(x^{\prime}\right) \sum_{j=1}^{n-1} a_{j n}\left(x^{\prime}, 0\right) \xi_{j}, b_{1}\left(x^{\prime}, \xi^{\prime}\right)=-i c\left(x^{\prime}\right) a_{n n}
$$

and according to (2.11),

$$
q\left(x^{\prime}, \xi^{\prime}\right)=d\left(x^{\prime}\right)-i c\left(x^{\prime}\right) \sum_{j=1}^{n-1} a_{j n} \xi_{j}-i c\left(x^{\prime}\right) a_{n n}\left(t^{\prime}+s\right) .
$$

Applying (3.19) we find that

$$
q(x, \xi)=-i c(x) A(x, \xi) / 2+d(x)-i c(x) a_{n n} s .
$$

(We have omitted the primes for convenience.)

It is natural to try to satisfy the conditions of Theorem 3.1 by setting $a(x)=c(x) / 2, e(x, \xi)=-i A(x, \xi)$ and $b(x, \xi)=d(x)-i c(x) a_{n n} s(x, \xi)$. By the root assumption $e(x, \xi)$ is (strongly) elliptic of order 1 and so (3.2) and (3.3) are fulfilled. If $c(x)$ never vanishes then $q$ is elliptic. If $c(x)$ has a simple zero, then $q$ cannot be hypoelliptic; we are then in a situation where the principal part determines the properties of the operator. If all the zeros of $c(x)$ are at least double, then (3.1) is also satisfied. Assumption (3.4) is always satisfied. Condition (3.5) is equivalent to

$$
|c(x)|+|d(x)| \geqslant c>0,
$$

and (3.6) is fulfilled (at least near the zeros of $c(x)$, where it counts) if $|\arg [c(x) / d(x)]| \leqslant \pi / 2-\epsilon$ for $\epsilon>0$. In particular we get the case discussed by [10] for $c(x), d(x) \geqslant 0, c(x)+d(x)=1$. In [2] existence and regularity are proved under the assumptions $c(x) \geqslant 0, c(x) \neq 0$,

$$
\frac{1}{2} \sum_{j=1}^{n} \cos \left(\nu, x_{j}\right)\left(\sum_{k=1}^{n} a_{j k} i D_{k} \varphi+b_{j} \varphi\right)+d(x)>0
$$

on the boundary, where $\nu$ is the outer normal and $\varphi$ is the solution, with Dirichlet data $\varphi=c(x)$ on the boundary, of a certain elliptic equation. According to the maximum principle $\varphi(x)>0$ in $G$. Hence if $c(x)=\varphi(x)=0$ at a certain point $x \in \partial G$ then $\operatorname{grad} \varphi(x)=\lambda \nu(x)$ (since the tangential derivatives have to 
vanish at $x$ ) with $\lambda \leqslant 0$, which implies that the sum in (3.22) is nonpositive there, so that $d(x)>0$. Thus we have hypoellipticity near the zeros of $c(x)$. (If $c(x) \neq 0$, then $q$ is elliptic.) Condition (3.22) is really nothing but a sufficient condition for the validity of the strong Gårding inequality for $q$; see [12].

Generally speaking, Theorem 3.1 provides us with a sufficient condition for hypoellipticity of certain boundary value problems. For if $q$ is given by (2.11) we can always try to find out whether $q$ belongs to a known class of hypoelliptic operators. If we can find $a, e$ and $b$ satisfying the condition of Theorem 3.1 with $q=a e+b$, then by Theorem 2.1 the boundary value problem is hypoelliptic.

REMARK. Example 2 suggests that condition (3.3) (which is certainly satisfied if $e$ is elliptic) is related to the occurrence of zeros of high multiplicity only in $\xi$. It was pointed out to the author by C. Fefferman that if $e$ is homogenous of degree 1 and on $|\xi|=1$ has only zeros of finite multiplicity greater than two, then (3.3) can be proved using the Weierstrass-Malgrange preparation theorem.

4. In this section we shall see how a more complete characterization of hypoelliptic boundary value problems can be given in the two-dimensional case. This characterization is based on an extension to pseudo-differential operators of the results established in [3] , [11] for ordinary differential operators.

Let $L=L(x, D)$ be an ordinary pseudo-differential operator of order $m$ defined in an open set $V \subset R$, with symbol $q(x, \xi)$ given by

$$
q(x, \xi) \sim \sum_{j=-\infty}^{m} q_{j}(x, \xi)
$$

where $q_{j}(x, \xi)$ is positively homogeneous of degree $j$. Using the fact that the sphere subbundle of the cotangent bundle is not connected in the one-dimensional case, and in fact can be written as $R \times\{t,-\}$, we can reduce the study of $q$ to the study of its restrictions to the positive and negative half lines (in $\xi$ ). In fact, letting $h(\xi) \in C^{\infty}(R), h(\xi)=0$ for $\xi<0$ and $h(\xi)=1$ for $\xi \geqslant 1$ and setting $H=$ $h(D)$, we can state

LEMMA 4.1. $L(x, D)$ is hypoelliptic in $V$ if and only if for every $u \in D^{\prime}(V)$,

$$
W F(u) \subset W F(L(x, D) H u) \cup(V \times\{-\})
$$

and

$$
W F(u) \subset W F(L(x, D)(I-H) u) \cup(V \times\{+\}) .
$$

(Here $W F(u)$ is the wave front set of $u$ as defined, e.g., in [9].)

Proof. If $L(x, D)$ is hypoelliptic, then sing supp $H u \subset \operatorname{sing} \operatorname{supp} L(x, D) H u$. 
But $W F(L(x, D) H u) \subset W F(H u) \subset V \times\{+\}$ implies

$$
\begin{aligned}
W F(H u) & =\operatorname{sing} \operatorname{supp} H u \times\{+\} \subset \operatorname{sing} \operatorname{supp} L(x, D) H u \times\{+\} \\
& =W F(L(x, D) H u)
\end{aligned}
$$

and similarly

$$
W F((I-H) u) \subset W F(L(x, D)(I-H) u)
$$

from which (4.2) and (4.3) follow.

Conversely, let $u \in D^{\prime}(V), L(x, D) u \in C^{\infty}$. Then $L H u=H L u+[L, H] u$ $\in C^{\infty}$ since $[L, H]$ is an operator of order $-\infty$, as its symbol is supported in a subset of $V \times \operatorname{supp} h^{\prime}(\xi) \subset V \times[0,1]$. Similarly $L(I-H) u \in C^{\infty}$. Hence $W F(u) \subset(V \times\{-\}) \cap(V \times\{+\})=\varnothing$ and $u \in C^{\infty}(V)$.

Lemma 4.1 enables us to restrict ourselves wl.o.g. to the case $q_{j}(x, \xi)=$ $a_{j}(x) \xi^{j}$ and to investigate whether (4.2) is valid or not. If $a_{m}(x) \neq 0$ then $L$ is elliptic near $x$. As in [3], [11] we restrict ourselves to the case where the zeros of $a_{m}(x)$ have all finite multiplicity and thus are isolated. (Note that the results of $\S 3$ are more general in this respect.) Let us assume that $0 \in V$, that $a_{m}(0)=$ 0 and that $a_{m}(x) \neq 0$ for $x \neq 0$. Let $n_{j}$ be the multiplicity of the zero of $a_{j}(x)$ at the origin $\left(n_{j}\right.$ may be equal to $+\infty$ for $j<m$ ). The sequence $\left\{n_{j}-j\right\}_{j=-\infty}^{m}$ is bounded below and thus the characteristic index of $L$ may be defined as the integer $m-r$ where $-\infty<r \leqslant m$ is specified by the requirements that $n_{r}-r=$ $\min \left\{n_{j}-j\right\}$ and $r=\max \left\{j: n_{j}-j=n_{r}-r\right\}$. We now claim that (4.2) cannot be true if $r=m$. In fact, we are in the case of regular singular point. Let

$$
a_{j}(x) \sim \sum_{k=n_{j}}^{\infty} a_{j, k} x^{k}
$$

be the formal Taylor expansion of $a_{j}(x)$ at the origin. We try a formal solution of $L(x, D) u=0$ of the form

$$
u(x) \sim x^{\lambda} \sum_{l=0}^{\infty} u_{l} x^{l}
$$

where $(i D)^{-j}$ is interpreted as mapping $x^{l}$ to $x^{l+j} /[(l+j)(l+j-1) \ldots(l+1)]$. We obtain an infinite system of linear equations of the form

$$
\sum_{k-j \leqslant l} C(j, k, l, \lambda) u_{l+j-k}=0, \quad l=n_{r}-r, n_{r}-r+1, \ldots
$$

where $C(j, k, l, \lambda)$ is a certain rational function of $j, k, l, \lambda$. The indicial equation for $\lambda$ is thus

$$
\sum_{k-j=n_{r}-r} C\left(j, k, n_{r}-r, \lambda\right)=\sum_{n_{j}-j=n_{r}-r} C\left(j, n_{j}, n_{r}-r, \lambda\right)=0 .
$$

Let $j_{0}=\min \left\{j: n_{j}-j=n_{r}-r\right\}$. Replacing $L(x, D)$ by $L(x, D) D^{-j_{0}}$ (if $\left.j_{0}<0\right)$ 
does not change (4.2) and has the effect of making the indicial equation a polynomial equation of degree $m-j_{0}=\operatorname{order}\left(L D^{-j_{0}}\right)$. We may thus assume that $j_{0} \geqslant 0$ and that the indicial equation has $m$ roots $\lambda_{1}, \ldots, \lambda_{m}$, repeated according to their multiplicities and ordered by $\operatorname{Re} \lambda_{1} \geqslant \operatorname{Re} \lambda_{2} \geqslant \ldots \geqslant \operatorname{Re} \lambda_{m}$. We now treat several cases separately, as in $[11, \S 5]$. If $\lambda_{1}$ is not an integer, let $x^{\lambda_{1}} \Sigma_{l=0}^{\infty} u_{l} x^{l}$ be the formal solution associated with it, let $u(x) \in C^{\infty}(V)$ be such that $l ! u^{(l)}(0)=u_{l}$ (such a function exists by Borel's theorem) and set

$$
v(x)=H\left(\lambda_{1}, x\right) u(x)
$$

where $H\left(\lambda_{1}, x\right)$ is the distribution defined in $\left[11\right.$, p. 119]. Then $L v \in C^{\infty}(V)$, but $W F(u) \supset\{0\} \times\{+\}$ (this follows from actual computation of the Fourier transform of $H(\alpha, x)$ as given e.g. in [1]). If $\lambda_{1}$ is a positive integer, $\lambda_{1} \geqslant r-1$, then we set

$$
\begin{array}{rlrl}
v(x) & =u(x) x^{\lambda_{1}}, & x \geqslant 0, \\
& =0, & & x<0,
\end{array}
$$

and once again $L v \in C^{\infty}(V)$ but $W F(v) \supset\{0\}+\{+\}$ since by differentiating $v$ to a sufficiently high degree we get $\delta(x) \bmod C^{\infty}$. For the other cases note that the distributions $R\left(x^{-k}\right), R\left(x^{j} \ln |x|^{n}\right)$ constructed in [11, p. 121] all have their wave front set equal to $\{0\} \times\{+,-\}$. Since if $\lambda_{1}$ is an integer $<r-1$ the formal solutions have to contain (as shown in [11, pp. 125-126]) $x^{-k}$ or $x^{j} \ln (x)^{n}$, we may construct in every case a distribution $v$ with $L v \in C^{\infty}(V)$ but $W F(u) \supset\{0\} \times\{+\}$ and then (4.2) (and (4.3)) cannot hold if $r=m$.

If $r<m$ we may assume (multiplying by a power of $D$ if necessary) that $r=0$. The analysis of this case is greatly facilitated by

Lemma 4.2. Let $m>r=0, a_{0}(x) \equiv 1$. Then there exists a pseudo-differential operator $C$ of order -1 and a differential operator $Q$ such that the operator $S=Q(I+C)^{-1}$ has the symbol $\Sigma_{j=-\infty}^{m} s_{j}(x) \xi^{j}$ where $s_{j}^{(l)}(0)=a_{j}^{(l)}(0)$, $-\infty<j \leqslant m, 0 \leqslant l<\infty$.

PRoof. Using the same letter for operators and their symbols, set $A=$ $\Sigma_{j=1}^{m} q_{j}(x, D), B=\Sigma_{j=-\infty}^{-1} q_{j}(x, D)$ (so that $L=A+I+B$ ). Denote by $N$ the projection on symbols of negative order,

$$
N\left(\sum_{j=-\infty}^{m} q_{j}(x, \xi)\right)=\sum_{j=-\infty}^{-1} q_{j}(x, \xi) .
$$

We want to solve the equation

$$
N(A+I+B)(I+C)=0
$$

or

$$
N(A+I+A C+B+C+B C)=N(A C)+B+C+B C=0 .
$$


Setting $-(I+B)^{-1} B=E$ we find that $E$ is also a pseudo-differential operator of order $\leqslant-1$, and (4.6) is equivalent to

$$
(I+B)^{-1} N(A C)+C=E .
$$

A formal solution of (4.7) is given by

$$
C=\sum_{j=0}^{\infty}(-1)^{j}\left[(I+B)^{-1} N A\right]^{i} E
$$

where $A$ and $(I+B)^{-1}$ are understood as multiplication operators on the ring of pseudo-differential operators. Writing formally

$$
\begin{aligned}
& E=\sum_{j=-\infty}^{-1} \sum_{k=0}^{\infty} e_{j, k} x^{k} \xi^{j}, C=\sum_{j=-\infty}^{-1} c_{j, k} x^{k} \xi^{j}, \\
& A=\sum_{j=1}^{m} \sum_{k=n_{j}}^{m} a_{j, k} x^{k} \xi^{j}
\end{aligned}
$$

we note that only finitely many terms in (4.8) contribute to any fixed $c_{j, k}$ upon equating coefficients of $x^{k} \xi^{j}$ in (4.8). Indeed, by assumptions $n_{j}-j>n_{r}-r$ $=0$ for $j \geqslant 0$. Hence if the inverse image under $(I+B)^{-1} N A$, of $x^{k} \xi^{j}$ contains a linear combination of terms of the form $x^{k_{1}} \xi^{j_{1}}$, then $k-j \geqslant k_{1}-j_{1}+1$. But $\operatorname{Im}\left((I+B)^{-1} N A\right)$ consists of operators of negative orders and order $E \leqslant$ -1 . Hence $\left[(I+B)^{-1} N A\right]^{-n} x^{k} \xi^{j}=\varnothing$ for $n>k-j$. Thus (4.8) gives a well-defined formula for $c_{j, k}$ in terms of $a_{j, k},-\infty<j \leqslant m$ (the symbols of $B$ and $E$ are determined by the symbol of $A)$. Let $c_{j}(x) \in C^{\infty}(V)$ be such that $c_{j}^{(k)}(0)=c_{j, k}, k=0,1, \ldots$, and let now $C$ be a pseudo-differential operator with symbol $c(x, \xi) \sim \Sigma_{j=-\infty}^{-1} c_{j}(x) \xi^{j}$. Then $I+C$ is elliptic and the pseudo-differential operator $Q^{\prime}=(A+I+B)(I+C)=L(I+C)$ has the symbol $\sum_{j=-\infty}^{m} r_{j}(x) \xi^{j}$ with $r_{j}^{(k)}(0)=0$ for $-\infty<j \leqslant-1,0 \leqslant k<\infty$. Setting $Q=$ $\sum_{j=0}^{m} r_{j}(x) D^{j}$, we obtain the lemma.

Turning our attention to differential operators $L$ of order $m$ where $r=0$ and $a_{0}(0) \neq 0$, we are led to the consideration of the determining factors $Q_{1}$, $\ldots, Q_{m}$ (see [3], [11] for their definitions and main properties). The analogue of Theorem 1 of [11] in this case is

THEOREM 4.3. $L$ satisfies (4.2) if and only if for every $1 \leqslant j \leqslant m$, either $\left|\operatorname{Re} Q_{j}(x)\right| \rightarrow \infty$ or $Q_{j}(x)$ is purely imaginary and $D Q_{j}(x) \rightarrow-\infty$, as $x \rightarrow 0$. $L$ satisfies (4.3) if and only if for every $1 \leqslant j \leqslant m$, either $\left|\operatorname{Re} Q_{j}(x)\right| \rightarrow \infty$ or $Q_{j}(x)$ is purely imaginary and $D Q_{j}(x) \rightarrow+\infty$, as $x \rightarrow 0$.

PROOF. It was shown in $[11, \mathrm{p} .114]$ that for every $f \in C^{\infty}(V)$ there exists a $v \in C^{\infty}(V)$ with $L v=f$. If $W F(L(x, D) H u)=\varnothing$ then setting $L(x, D) H u$ $=f$ we obtain $L(x, D)(H u-v)=0, W F(H u-v)=W F(H u)$. Hence (4.2) will 
follow once we prove that for all distributions $u$ satisfying $L u=0, W F(u) \subset$ $\{0\} \times\{-\}$ (note that $L$ is elliptic in $\backslash\{0\}$ ). All such solutions $u$ are of the form $u_{j}(x)=\exp Q_{j}(x) \cdot v_{j}(x) x^{\rho} j$ where $v_{j}(x)$ is a (formal) log-fractional powers sum (see (1.2) and (1.3) in [11]). It is proved in [11] that all distributions $u_{j}$ corresponding to $\operatorname{Re} Q_{j}(x) \rightarrow-\infty$ are actually smooth, and that no distribution solution corresponds to $\operatorname{Re} Q_{j}(x) \rightarrow+\infty$. The purely imaginary determining vectors are dealt with in

LeMma 4.4. Let $\rho$ be a complex number, let $b_{1}<\ldots<b_{k}, c_{1}, \ldots, c_{k}$ be real numbers, $c_{k}>0$, and let $n$ be an integer. Then the distribution $u \in$ $D^{\prime}(R)$, supp $u \subset[0, \infty)$ obtained by regularizing the function

$$
x^{\rho}(\ln x)^{n} \exp \left(i \sum_{j=1}^{k} c_{j} x^{-b_{j}}\right)
$$

has the property $W F(u)=\{0\} \times\{-\}$, and the distribution vobtained similarly from

$$
x^{\rho}(\ln x)^{n} \exp \left(-i \sum_{j=1}^{k} c_{j}|x|^{-b_{j}}\right)
$$

satisfies $W F(v)=\{0\} \times\{+\}$.

Proof Of Lemma 4.4. Let $0<\epsilon<1$ be any number. The function $u(z)=z^{\rho}(\ln z)^{n} \exp \left(i z^{-\epsilon}\right)$ can be defined holomorphically in the half plane $\operatorname{Im} z<0$ in such a way that $z^{-\epsilon}$ is the branch which is positive for $x \rightarrow 0$ with cut at the negative axis. For that branch $\operatorname{Re} i z^{-\epsilon} \leqslant 0$ for $\operatorname{Im} z<0$ and $\operatorname{Re} i(x-i 0)^{-\epsilon} \rightarrow-\infty$ as $x>0$. Thus the distribution $w$ obtained as the limit as $y \rightarrow 0_{-}$of $u(x+i y)$ satisfies $W F(w)=\{0\} \times\{-\}$, and the restriction of $w$ to the negative axis is a $C^{\infty}$ function with $w^{(l)}(x) \rightarrow 0$ as $x \rightarrow 0$. Hence $u-w \in C^{\infty}\left(R^{1}\right)$ which proves the assertion in this case if $\operatorname{Re} \rho \geqslant 0$ (the case $\operatorname{Re} \rho<0$ follows from this by differentiation). Let now $\varphi \in C_{0}^{\infty}\left(R^{1}\right), \varphi \equiv 1$ near $x=0$. We shall prove that the Fourier transform of $\varphi(x) \exp \left[i \Sigma_{j=1}^{k} c_{j} x^{-b_{j}}\right] H(x)$ decreases rapidly as $\xi \rightarrow \infty$. It would follow then that

$$
W F\left(\exp \left(i \sum_{j=1}^{k} c_{j} x^{-b_{j}}\right) H(x)\right)=\{0\} \times\{-\}
$$

(since sing supp $\exp [i \Sigma \ldots]=\{0\})$. The lemma would thus follow from the formula $W F\left(u_{1} u_{2}\right) \subset W F\left(u_{1}\right) \cup W F\left(u_{2}\right) \cup\left(W F\left(u_{1}\right)+W F\left(u_{2}\right)\right)$ proved in [9], where

$$
\begin{aligned}
& u_{1}=x^{\rho}(\ln x)^{n} \exp \left[i x^{-\epsilon}\right], \\
& u_{2}=\exp \left[-i x^{-\epsilon}+i \sum_{j=1}^{k} c_{j} x^{-b_{j}}\right] \text { for } 0<\epsilon<b_{1} .
\end{aligned}
$$


(The second half of the lemma would follow in a similar way.)

We integrate

$$
I(\xi)=\int_{0}^{\infty} \varphi(x) \exp \left[i \sum_{j=1}^{k} c_{j} x^{-b_{j}}-i x \xi\right] d x
$$

by parts as follows $(\xi>0)$ :

$$
\begin{aligned}
& I(\xi)= \int_{0}^{\infty} \exp \left[i\left(c_{k} x^{-b_{k}}-x \xi\right)\right] \varphi(x) \exp \left[i \sum_{j=1}^{k-1} c_{j} x^{-b_{j}}\right] d x \\
&= i \int_{0}^{\infty}\left\{(d / d x) \exp \left[i\left(c_{k} x^{-b_{k}}-x \xi\right)\right]\right\} \\
& \cdot\left(c_{k} b_{k} x^{-b_{k}-1}+\xi\right)^{-1} \cdot \varphi(x) \exp \left[i \sum_{j=1}^{k-1} c_{j} x^{-b_{j}}\right] d x \\
&(4.10)=-i \int_{0}^{\infty} \exp \left[i\left(c_{k} x^{-b_{k}}-x \xi\right)\right](d / d x) \cdot\left(c_{k} b_{k} x^{-b_{k}-1}+\xi\right)^{-1} \varphi(x) \exp \left[i \sum_{j=1}^{k-1} c_{j} x^{-b}\right] d x=\ldots \\
&=(-i)^{N} \int_{0}^{\infty} \exp \left[i\left(c_{k} x^{-b_{k}}-x \xi\right)\right] \\
& \quad \cdot\left\{(d / d x)\left(c_{k} b_{k} x^{-b_{k}-1}+\xi\right)^{-1}\right\}^{N} \cdot \varphi(x) \exp \left[i \sum_{j=1}^{k-1} c_{j} x^{-b_{j}}\right] d x .
\end{aligned}
$$

In order to justify these integrations, observe that

$$
\left(c_{k} b_{k} x^{-b_{k}-1}+\xi\right)^{-1}=d_{k} x^{b_{k}+1}\left(1+d_{k} x^{b_{k}+1} \xi\right)^{-1}
$$

(where $d_{k}=\left(c_{k} b_{k}\right)^{-1}>0$ ). Hence each application of the operator $A=$ $(d / d x)\left(c_{k} b_{k} x^{-b_{k}-1}+\xi\right)^{-1}$ to an expression of the form $x^{\lambda} \exp \left[i \Sigma_{j=1}^{k-1} c_{j} x^{-b_{j}}\right]$ yields a sum of terms where $x^{\lambda}$ is either replaced by $x$ raised to a power $\geqslant \lambda+$ $b_{k}$, or by $x$ to the power $\lambda+b_{k}+1-b_{j}-1>\lambda, 1 \leqslant j \leqslant k-1$. Since we started with $\lambda=0$, we find that the right-hand side of (4.10) is integrable for each $N$. Set now $x \xi^{1 /\left(1+b_{k}\right)}=y$. Then

$$
A=d_{k} \xi^{-b_{k} /\left(1+b_{k}\right)}(d / d y) y^{b_{k}+1}\left(1+d_{k} y^{b_{k}+1}\right)^{-1}
$$

and

$$
\begin{aligned}
& U(\xi) \mid \leqslant d_{k}^{N} \xi^{-1 /\left(b_{k}+1\right)-N b_{k} /\left(1+b_{k}\right)} \\
& \cdot \int_{0}^{\infty} \mid\left[(d / d y) y^{b_{k}+1}\left(1+d_{k} y^{b_{k}+1}\right)^{-1}\right]^{N} \varphi\left(y \xi^{-1\left(1+b_{k}\right)}\right) \\
& \cdot \exp \left[i \sum_{j=1}^{k-1} c_{j} \xi^{b_{j} /\left(1+b_{k}\right)} y^{-b_{j}}\right] \mid d y .
\end{aligned}
$$


Every differentiation of the exponent in the right-hand side can add a factor less than or equal to $\xi^{b_{k-1} /\left(1+b_{k}\right)}$. Thus we have (with a constant $C_{N}$ depending only on $\left.b_{1}, \ldots, b_{k}, d_{k}, c_{1}, \ldots, c_{k-1}\right)$ that

$$
|I(\xi)| \leqslant C_{N} \xi^{N\left(b_{k-1}-b_{k}\right) /\left(1+b_{k}\right)} .
$$

But $b_{k-1}<b_{k}$. Hence $I(\xi)=O\left(\xi^{-N}\right)$ as $\xi \rightarrow \infty$ for all $N$.

END OF THE Proof of TheOREM 4.3. Let now $Q_{j}(x)$ be a purely imaginary determining factor, $Q_{j}(x)=i \Sigma_{t=1}^{k} c_{t} x^{-b_{t}}, 0<b_{1}<\ldots<b_{t}$. Then $D_{x} Q_{j}(x) \rightarrow-\infty$ if and only if $c_{k}>0$. If $c_{k}>0$ and $u_{j}(x)$ is a corresponding solution of $L u_{j}=0$, then by Lemma $4.4 W F\left(u_{j}\right)=\{0\} \times\{-\}$. If, however, $c_{k}<$ 0 we can multiply every term in the formal expansion for $v_{j}$ by a suitable cutoff function equal to 1 near $x=0$ so as to obtain a distribution $w$ having the same expansion near the origin, and $L\left(w x^{\rho_{j}} \exp Q_{j}(x)\right) \in C^{\infty}(V)$, but $W F\left(w x^{\rho_{j}} \exp Q_{j}(x)\right)=\{0\} \times\{+\}$ by the second part of Lemma 4.4. The second statement in Theorem 4.3 is proved similarly.

We may now state and prove the main theorem of this section.

THEOREM 4.5. Let $L(x, D)$ be an ordinary pseudo-differential operator with symbol given by

$$
\sum_{j=-\infty}^{m}\left[a_{j}^{+}(x) \xi^{j} h(\xi)+a_{j}^{-}(x) \xi^{j} h(-\xi)\right]
$$

where $a_{j}^{+}, a_{j}^{-} \in C^{\infty}(V)$ and $h(\xi) \in C^{\infty}\left(R^{1}\right)$ satisfies $h(\xi)=1$ for $\xi>1, h(\xi)=0$ for $\xi \leqslant 0$. Let $L^{+}\left(L^{-}\right)$be a pseudo-differential operator having the symbol

$$
\sum_{j=-\infty}^{m} a_{j}^{+}(x) \xi^{j}\left(\sum_{j=-\infty}^{m} a_{j}^{-}(x) \xi^{j}\right) .
$$

Let $\mathrm{m}^{+}\left(\mathrm{m}^{-}\right)$and $\mathrm{r}^{+}\left(\mathrm{r}^{-}\right)$denote the orders and characteristic indices of $\mathrm{L}^{+}$ $\left(L^{-}\right)$respectively. Suppose that $a_{m+}$ and $a_{m_{-}}$have a zero of a finite multiplicity in $0 \in V, a_{m^{+}}(x) \neq 0, a_{m-}(x) \neq 0$ for $x \in V \backslash\{0\}$. Then a necessary condition for the hypoellipticity of $L$ is that $a_{r^{+}}(0) \neq 0$ and $a_{r^{-}}(0) \neq 0$. Let $C^{+}\left(C^{-}\right)$ be a pseudo-differential operator of order $-1, Q^{+}\left(Q^{-}\right)$be a differential operator of order $m^{+}-r^{+}\left(m^{-}-r^{-}\right)$such that the operator

$$
T^{+}=a_{r^{+}}(x) Q^{+}\left(I+C^{+}\right)^{-1} \xi^{+} \quad\left(T^{-}=a_{r^{-}}(x) Q^{-}\left(I+C^{-}\right)^{-1} \xi^{r^{-}}\right)
$$

has the symbol

$$
\sum_{j=-\infty}^{m} s_{j}^{+}(x) \xi^{j}\left(\sum_{j=-\infty}^{m^{-}} s_{j}^{-}(x) \xi^{j}\right)
$$

satisfying $s_{j}^{+(l)}(0)=a_{j}^{+(l)}(0) \quad\left(s_{j}^{-(l)}(0)=a_{j}^{-(l)}(0)\right)$ for $-\infty<j \leqslant m^{+} \quad(-\infty<$ $\left.j \leqslant m^{-}\right), 0 \leqslant l<\infty$. (Such operators exist by Lemma 4.2). Let $Q_{1}^{+}, \ldots$, $Q_{m^{+}-r^{+}}^{+}\left(Q_{1}^{-}, \ldots, Q_{m_{--r^{-}}^{-}}\right)$be the determining factors of $T^{+}\left(T^{-}\right)$. Then 
$L$ is hypoelliptic if and only if for every $1 \leqslant j \leqslant m^{+}-r^{+}$, either $\left|\operatorname{Re} Q_{j}^{+}(x)\right|$ $\rightarrow \infty$ or $Q_{j}^{+}(x)$ is purely imaginary and $D_{x} Q_{j}^{+}(x) \rightarrow-\infty$, as $x \rightarrow 0$, and for every $1 \leqslant j \leqslant m^{-}-r^{-}$, either $\left|\operatorname{Re} Q_{j}^{-}(x)\right| \rightarrow-\infty$ or $Q_{j}^{-}(x)$ is purely imaginary and $D_{x} Q_{j}^{-}(x) \rightarrow+\infty$, as $x \rightarrow 0$.

Proof of Theorem 4.5. Theorem 4.3 and Lemma 4.1 imply that the operator $M=T^{+} H+T^{-}(I-H)$ is hypoelliptic if and only if the conditions of Theorem 4.5 are fulfilled. The necessity of the conditions $a_{r}+(0) \neq 0, a_{r^{-}}(0)$ $\neq 0$ is proved as in $[11, \S 5]$. Noting that Lemma 3.1 of [11] remains true for pseudo-differential operators and that the homogeneous terms in the symbols of $L$ and $M$ possess the same formal Taylor expansion at the origin, we obtain that $L$ is hypoelliptic if and only if $M$ is.

The determining factors of differential operators are effectively computable from their coefficients. The coefficients of $Q^{+}$and $Q^{-}$are computable from finitely many terms of the expansion of the symbol of $L$. Hence the hypoellipticity of $L$ can be determined by inspecting a sufficiently large number of terms in the symbol of $L$. While the actual computation of the determining factors is not easy, the methods of [3] and [11] are applicable. We leave it to the reader to state the appropriate generalizations of the Theorems in [3] and [11]; we shall give instead two simple examples.

EXAMPLE 1. $q(x, \xi)=x^{2}|\xi|+1$. (This is of course well known and is included here only to illustrate the method.) For $\xi \rightarrow+\infty$ consider $x^{2} \xi+1$. Then $\xi=-1 /\left(x^{2}\right) \rightarrow-\infty$ and thus $D_{x} Q \rightarrow-\infty$. For $\xi \rightarrow-\infty$ consider $-x^{2} \xi+1$. Then $\xi=1 /\left(x^{2}\right)$ and $D_{x} Q \rightarrow+\infty$. Hence the hypoellipticity.

EXAmple 2. $q(x, \xi)=x^{4}|\xi|-1+i x^{2}$. This one is also hypoelliptic, but does not seem to be included in the usual classes of hypoelliptic pseudo-differential operators (compare [11, p. 108]). It follows that the following boundary value problem: $\Delta u=f$ in $y>0, x^{4} \partial u / \partial n-u+i x^{2} u=g$ in $y=0$ is hypoelliptic. This case is not covered by Theorem 3.1.

More generally, Theorem 4.5 enables us to test the hypoellipticity of many boundary value problems for second order equations in two variables (satisfying the root condition); all one has to do is to check whether or not the conditions of Theorem 4.5 are satisfied by $q$ as given in (2.11).

\section{REFERENCES}

1. I. M. Gel'fand and G. E. Šilov, Generalized functions. Vol. I: Properties and operations, Fizmatgiz, Moscow, 1958; English transl., Academic Press, New York, 1964. MR 20 \#4182; 29 \#3869.

2. K. Hayashida, On the singular boundary value problem for elliptic equations, Trans. Amer. Math. Soc. 184 (1973), 205-221. MR 48 \#6662.

3. B. Helffer and Y. Kannai, Determining factors and hypoellipticity of ordinary differential operators with double "characteristics," Asterisque 2/3 (1973), 197-216. 
4. L.Hörmander, On the regularity of the solutions of boundary problems, Acta. Math. 99 (1958), 225-264. MR 24 \#A1503.

5. Linear partial differential operators, Die Grundlehren der math. Wissenschaften, Band 116, Academic Press, New York; Springer-Verlag, Berlin, 1963. MR 28 \#4221.

6. Pseudo-differential operators and non-elliptic boundary value problems, Ann. of Math. (2) 83 (1966), 129-209. MR 38 \#1387.

7. Pseudo-differential operators and hypoelliptic equations, Proc. Sympos. Pure Math., vol. 10, Amer. Math. Soc., Providence, R. I., 1967, pp. 138-183.

8. On the index of pseudo-differential operators, Schriftenreihe Inst. Math. Deutsch. Akad. Wiss. Berlin Reihe A 8 (1969), 127-146.

9. - On the existence and the regularity of solutions of linear pseudo-differential equations, Enseignment Math. (2) 17 (1971), 99-163. MR 48 \#9458.

10. S. Ito, Fundamental solutions of parabolic equations and boundary value problems, Japan J. Math. 27 (1957), 55-102. MR 20 \#4702.

11. Y. Kannai, Hypoelliptic ordinary differential operators, Israel J. Math. 13 (1972), 106-134. MR 49 \#10951.

12. A. Melin, Lower bounds for pseudo-differential operators, Ark. Mat. 9 (1971), 117-140. MR 48 \#6735.

13. L. Nirenberg and F. Trèves, Solvability of a first order linear partial differential equation, Comm. Pure Appl. Math. 16 (1963), 331-351; MR 29 \#348.

14. R. T. Seeley, Singular integrals and boundary value problems, Amer. J. Math. 88 (1966), 781-809. MR 35 \#810.

DEPARTMENT OF MATHEMATICS, NORTHWESTERN UNIVERSITY, EVANSTON, ILLINOIS 60201

Current address: The Weizmann Institute of Science, Rehovot, Israel 